\title{
On the Effective Interaction of two Hydrogen Centres in Niobium*
}

\author{
P. Frodl ${ }^{1}$, F. T. Sommer ${ }^{2}$, K. Hau, and F. Wahl \\ Institut für Theoretische Physik, University Tübingen
}

Z. Naturforsch. 45a, 857-866 (1990); received April 6, 1990

\begin{abstract}
We derive an effective interaction between hydrogen impurities in Niobium using a microscopic theory of hydrogen in metals. Our model consists of an infinite bcc-crystal with two hydrogen centres occupying tetrahedral interstitial sites, neighbouring on the first to the fourth coordination shell. The elastic interactions are assumed to obey the classical harmonic approximation. The electronic interactions due to both the coulomb potentials and the overlap of the impurity induced electron densities in the vicinity of the interstitials also play an important role. These latter interactions are treated as two-body interactions in a zeroth order approximation of the New Tamm-Dancoffmethod. A separation ansatz results in an effective interaction which depends on the distance between the interstitials and upon the spin states of their excess electrons. We propose some improvements on our model, and to test our calculations, we construct the grand partition function of an appropriate lattice gas model.
\end{abstract}

Key words: Metal Hydrogen Systems - Microscopic Theory - H-Impurities - Electronic Interactions - Effective Potentials

\section{Introduction}

For many years research on hydrogen in metals has attracted the attention of physicists. The motivation for this has come from both a basic as well as an applied point of view [1]. In particular there are two topics of great interest: on the one hand the diffusional properties in metals of $\mathrm{H}$ and its isotopes allow a test of quantum theories of diffusion of light particles $[2,3]$; on the other hand calculated phase diagrams of metal-hydrogen systems should link microscopically motivated models to the experimentally observed macroscopic behavior [4].

The lattice formed by the interstitial sites of the metal crystal may be occupied or left vacant by the impurity $\mathrm{H}$ atoms. This forms a natural realization of the lattice gas model well known from statistical mechanics [4]. To employ the lattice gas model one must know the effective interactions between the hydrogen centres, which are localized at the interstitial sites. The importance of the long range attractive elastic interactions for mean-field-like phase diagrams has been formerly recognized [5] and has been investigated in de-

* Work supported by the Alfried Krupp von Bohlen und Halbach-Foundation.

1 Present address: Institut für Physik, Johannes GutenbergUniversität, Staudinger Weg 7, D-6500 Mainz 1.

2 Present address: C. u. O.-Vogt-Institut für Hirnforschung, Universitätsstr. 1, D-4000 Düsseldorf 1.

Reprint requests to Dr. P. Frodl, Institut für Physik, Johannes Gutenberg-Universität Mainz, Staudinger Weg 7, D-6500 Mainz, FRG. tail $[6,7]$. As the mean distance between the occupied interstitial sites becomes small at high storage densities, the specific form of the short range electronic interactions becomes important. While very crude approximations, (e.g., hardcore potentials [6] or screened coulomb potentials [8]) are already sufficient to reproduce the quantitative structure of the gas-liquid transition, a detailed knowledge of the intractions is necessary to understand the structure of ordered phases and saturation phenomena. It would therefore be desirable not only to have phenomenological model potentials, but also to have the means to calculate these interactions from a microscopic "first principles" theory.

Such a microscopic theory was introduced by Wahl et al. [9] using the New Tamm-Dancoff-method, which was originally applied to the study of elementary particles [10]. The main purpose of this method is to calculate the impurity induced energy changes in the elecronic system of the host crystal. Employing this theory we have recently treated a single hydrogen center in an infinite bcc-crystal as a model for dilute $\mathrm{H}$ in niobium. We calculated the lattice relaxation, the dipole force tensor, the relative change of volume and the heat of solution [11] as well as local excitation energies of the H-impurity [12]. The results of these calculations are in good agreement with the experimental data.

This paper presents the first attempt to study higher storage densities (i.e., the regime where the assump-

0932-0784 / 90 / 0700-0857 \$ 01.30/0. - Please order a reprint rather than making your own copy. 
tion of non-interacting impurities no longer holds). Our principle focus is the calculation of an effective interaction potential between neighbouring $\mathrm{H}$-centres in niobium. In contrast to other workers, who treat similar problems using density functional theories [13] or effective medium theories [14], we explicitely account for the dependence of the electronic energies on the host relaxation in the vicinity of the interstitials.

In Sect. 2 we introduce our model and define such basic concepts as storage energy, electronic and elastic energy and interaction energy. In Sect. 3 we proceed to study within the classical harmonic approximation, the lattice statics of tetrahedral interstitials neighbouring on the first four shells. Section 4 deals exclusively with the impurity induced perturbative forces and interaction energies due to both the proton and electron systems. We present our results in Section 5: Here we compare the storage energy of two H-centres in several configurations with twice the storage energy of one H-center. These configurations consist of neighbours of first, second, third and fourth order on the lattice of the interstitial sites. The comparison yields an effective interaction energy between the impurities. These interactions will be shown to depend on the distance between the interstitial protons and on the spin states of their excess electrons. Finally we discuss the shortcomings of our model and propose some improvements on it. We consider a modified lattice gas model which allows to derive experimentally measurable macroscopic quantities for tests of our calculations.

\section{A Microscopic Model Concerning Static Problems}

As with our preceeding papers on the static properties of metal hydrides $[9,11]$ we consider both the protons and the ions of the infinite $\mathrm{Nb}$ host lattice as classical pointlike charged particles. This approximation is justified by a twofold adiabatic decoupling: first of the electronic from the protonic and ionic subsystems and secondly the protonic from the ionic subsystem in both the hydrided and the unloaded crystal. Furthermore we are interested only in the equilibrium positions $\tilde{\boldsymbol{X}}$ of the host lattice. The protons are fixed at their ideal interstitial sites $\boldsymbol{Y}$. These approximations amount to a neglect of the vibrations and diffusive motion. The electrons of the host and the stored $\mathrm{H}$-atoms are treated quantum mechanically and are assumed to be in their ground states*.

* For details see [15].
The storage energy of $N$ hydrogen centres is defined as the ground state energy $E^{(N)}(\tilde{X}, \boldsymbol{Y})$ of the crystal with relaxed ion positions $\tilde{X}$ storing $N$ H-centres minus the sum of the energy $E\left(\boldsymbol{X}^{0}\right)$ of the unperturbed host crystal ( $\boldsymbol{X}^{0}$ represents the ions at their ideal lattice positions) and the ground state energy of $N$ non-interacting $\mathrm{H}$-atoms $(-N$ Ryd $)$. Formally we may write

$$
\begin{aligned}
\Delta E_{S}^{(N)}(\tilde{\boldsymbol{X}}, \boldsymbol{Y}) \stackrel{\text { def }}{=} & E^{(N)}(\tilde{\boldsymbol{X}}, \boldsymbol{Y})-E\left(\boldsymbol{X}^{\mathbf{0}}\right)+N \mathrm{Ryd} \\
& =\Delta E^{(N)}(\tilde{\boldsymbol{X}}, \boldsymbol{Y})+N \text { Ryd } .
\end{aligned}
$$

We define the interaction energy of $\mathrm{N} \mathrm{H}$-atoms introduced into the host crystal as the difference between the storage energy of $N \mathrm{H}$-centres embedded in the host and $N$ times the storage energy of a single $\mathrm{H}$-centre,

$$
W^{(N)}(\tilde{X}, \boldsymbol{Y}) \stackrel{\text { def }}{=} \Delta E_{S}^{(N)}(\tilde{X}, \boldsymbol{Y})-N \cdot \Delta E_{S}^{(1)}(\tilde{X}, \boldsymbol{Y})
$$

Most of the statistical theories of phase transitions deal with two-body interactions. We therefore restrict our calculations to the special case of $N=2$.

The energy difference $\Delta E^{(2)}(\tilde{X}, Y)$ between the hydride and the unperturbed crystal is divided into an elastic and an electronic contribution as

$$
\Delta E^{(2)}(\tilde{\boldsymbol{X}}, \boldsymbol{Y})=U_{\mathbf{L}}(\tilde{\boldsymbol{X}})+Q(\tilde{\boldsymbol{X}}, \boldsymbol{Y}) .
$$

The elastic contribution $U_{\mathrm{L}}$ represents the energy change of the unloaded host due to the displacements $\boldsymbol{s}=\tilde{\boldsymbol{X}}-\boldsymbol{X}^{\mathbf{0}}$ of the ions to their new equilibrium positions. We assume the form of a harmonic approximation,

$$
U_{\mathrm{L}}(\tilde{\boldsymbol{X}})=\frac{1}{2} \boldsymbol{s} \boldsymbol{\phi} \boldsymbol{s} ; \boldsymbol{\phi} \text { tensor of force constants. }
$$

The electronic contribution $Q$ includes the interactions between the stored protons and electrons assuming the host to be in the relaxed state,

$$
Q(\tilde{\boldsymbol{X}}, \boldsymbol{Y})=\sum_{n} \sum_{k=1}^{2} \frac{Z e^{2}}{\left|\tilde{\boldsymbol{X}}_{n}-\boldsymbol{Y}_{k}\right|}+\omega^{(2)}(\tilde{\boldsymbol{X}}, \boldsymbol{Y})+\frac{e^{2}}{\left|\boldsymbol{Y}_{1}-\boldsymbol{Y}_{2}\right|}
$$

$Z$ denotes the charge of the ions and

$$
\omega^{(2)}(\tilde{\boldsymbol{X}}, \boldsymbol{Y})=\varepsilon^{(z+2)}(\tilde{\boldsymbol{X}}, \boldsymbol{Y})-\varepsilon^{(z)}(\tilde{\boldsymbol{X}})
$$

is the energy difference between the $z+2$-electron system of the relaxed host storing $2 \mathrm{H}$-centres (the hydride system) and the $z$-electron system of a crystal with the ions at the equilibrium positions of the hydride system but without any implemented $\mathrm{H}$-atom (the reference system).

The equilibrium positions of the ions of the hydride system are obtained by minimizing the storage energy 


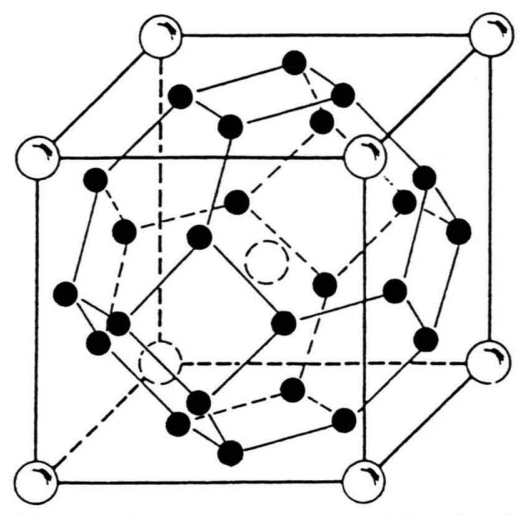

Fig. 1. The tetrahedral interstitial sites in a conventional bcc unit cell.

(1) with respect to the displacements $s$ of an appropriate number of ions in the vicinity of the interstitials

$$
\left.\nabla_{s_{m}} \Delta E_{s}^{(2)}(s, Y)\right|_{s_{0}}=0, \forall_{m} .
$$

This yields a system of force equations

$$
\sum_{n} \phi_{m n} s_{n}=-\nabla_{s_{m}} Q(s, Y)
$$

with the internal elastic forces $-\boldsymbol{F}_{\boldsymbol{m}}$ on the left side and the perturbative forces $f_{m}$ on the right side. Using the static Green's tensor $\mathscr{G}$ which obeys $\mathscr{G} \phi=\phi \mathscr{G}=1$, (7) can be inverted and resolved with respect to the displacement as

$$
s_{k}=\sum_{m} G_{k m} f_{m}(s, Y)
$$

Once the functional dependence of the perturbative forces on the displacements is known, (7) can be solved for arbitrary configurations of two neighbouring $\mathrm{H}$-centres on the interstitial lattice. Due to the symmetry group $\mathrm{O}_{\mathrm{h}}$ of the bcc-lattice, the tensors $G_{k m}$ reduce to only a few independent elements [15]. We have used the values of the matrix elements found in reference [16].

\section{Lattice Statics of two Neighbouring Hydrogen Impurities}

It is known from both experimental [17] and theoretical [18] investigations that in their ground state, hydrogen centres in niobium occupy the tetrahedral interstitial sites (Figure 1). As all interstitial sites are equivalent with respect to the host lattice, we may choose any one of them as representing the centre of the coordination
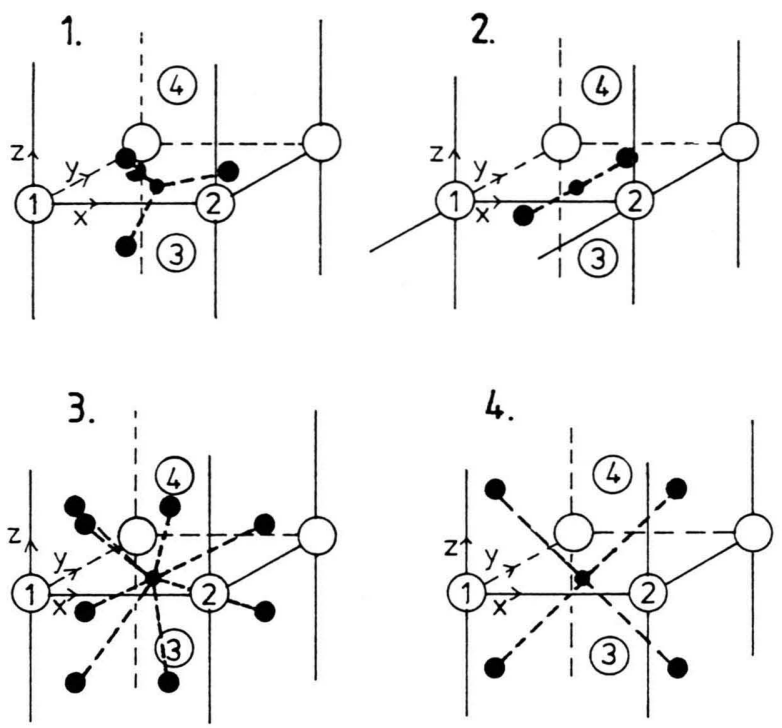

Fig. 2. Interstitial sites on the first four shells around one H-centre.

Table 1. Interstitial sites on the first four shells around the interstitial $\frac{a}{4}(2,1,0)$

\begin{tabular}{llll}
\hline Shell & Distance & Coordinates $\left[\frac{a}{4}\right]$ & $\begin{array}{l}\text { Represen- } \\
\text { tative }\end{array}$ \\
\hline 1 & $\frac{a}{4} \sqrt{2}$ & $(2,0,1),(2,0,-1)$ & $(3,2,0)$ \\
& $1.17 \AA$ & $(1,2,0),(3,2,0)$ & \\
2 & $\frac{a}{4}$ & $(2,-1,0),(2,3,0)$ & $(2,3,0)$ \\
& $1.65 \AA$ & & \\
3 & $\frac{a}{4} \sqrt{6}$ & $(4,2,1),(4,2,-1)$ & \\
& $2.02 \AA$ & $(3,0,2),(3,0,-2)$ & $(3,0,2)$ \\
& & $(0,2,1),(0,2,-1)$ & \\
4 & $\frac{a}{2} \sqrt{2}$ & $(4,1,2),(4,1,-2)$ & $(4,1,2)$ \\
& $2.33 \AA$ & $(0,1,2),(0,1,-2)$ & \\
\hline
\end{tabular}

shells on the lattice of interstitials. Previous models employing hard-core short range electronic interactions have been motivated by the structure of the ordered $\beta$-phase. This phase exhibits no occupation up to the third shell around a given hydrogen impurity [17]. Our aim is to calculate an interaction potential within this "hard-core"-region, therefore we only consider neighbouring $\mathrm{H}$-centres on the first four shells (Fig. 2 and Table 1). As all interstitials on shells up to the seventh are equivalent with respect to the 
1.
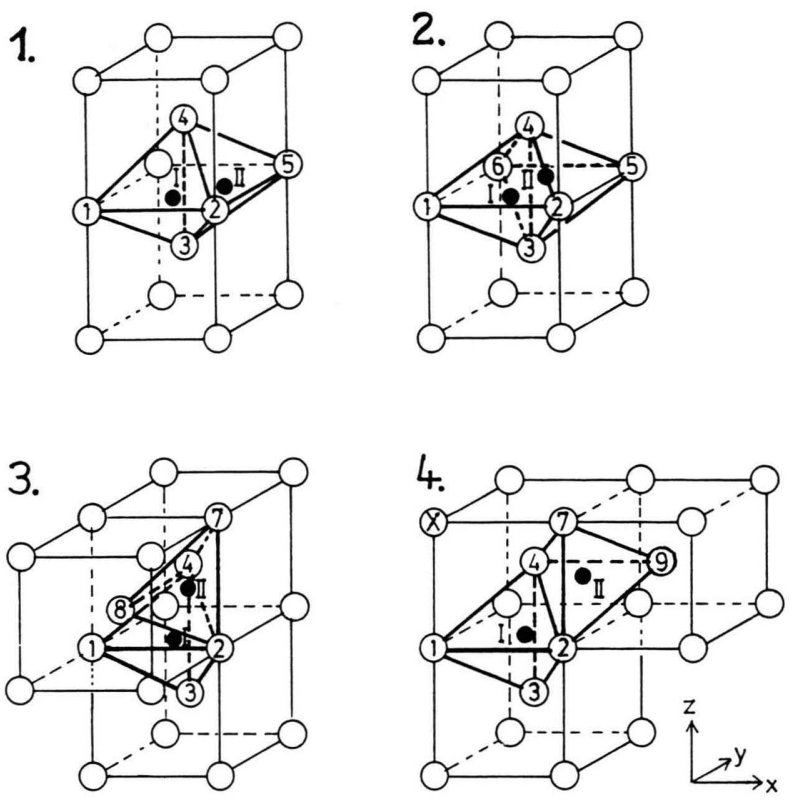

Fig. 3. Four configurations of neighbouring H-centres I and II and the ions participating in the relaxation.

centre of the shells, we can restrict our investigations to a single representative of each. In particular we consider the four configurations with two occupied interstitials,

$$
\begin{array}{ll}
\text { configuration 1: } & \frac{a}{4}(2,1,0)-\frac{a}{4}(3,2,0), \\
\text { configuration 2: } & \frac{a}{4}(2,1,0)-\frac{a}{4}(2,3,0), \\
\text { configuration } 3: & \frac{a}{4}(2,1,0)-\frac{a}{4}(3,0,2), \\
\text { configuration } 4: & \frac{a}{4}(2,1,0)-\frac{a}{4}(4,1,2) .
\end{array}
$$

Here $a$ denotes the lattice constant of $\mathrm{Nb}(3.30 \AA)$ and the origin of the reference frame is situated at ion 1 (see Figure 3).

In our calculations external perturbative forces and ionic displacements are confined to the nearest neighbours of the impurities. We justify this approximation by the encouraging results of our previous investigations [11] and certain experiments [19] which show the displacement of the next nearest ions to be considerably smaller to that of the nearest neighbours. We further assume the perturbative forces to be additive central forces depending only on the distance between the impurities and the host ions. Due to the smallness of the non-radial components of the displacements, we also suppose that they act only along the direction from the impurity to the unrelaxed ion.

Using these assumptions, we extract from (8) a system of equations that specify a given configuration,

$$
\boldsymbol{s}=g \boldsymbol{f}_{\mathrm{rad}}(\boldsymbol{s}) \text {. }
$$

$\boldsymbol{s}$ denotes the $3 \mathrm{~K}$-dimensional vector of the displacements of the $\mathrm{K}$ ions involved. $\mathrm{g}$ is a reduced Green's matrix determining the response of these ions to the forces acting upon them. $f_{\text {rad }}$ is a vector, which is a function only of the $\mathrm{K}$ absolute values of these forces. The functional dependence on both the directions of these forces, and the forces exerted on the ions due to the pair of impurities, is contained implicitely in $g$. Thus $g$ is a $\mathrm{K} \times 3 \mathrm{~K}$-matrix. For the sake of clarity Fig. 3 shows a compilation of the four configurations where each of the relevant ion is denoted by a number.

As discussed above the equivalence of the interstitial sites with respect to the host lattice allows us to restrict our calculations to the vicinity of one of the two impurities. The effect of the second impurity is taken into account via the reduced Green's matrices $g$. We than infer the displacements of the ions neighbouring the second impurity from the symmetry properties of the bond. Thus (9) reduces to 7 (configuration 1), 3 (conf. 2), 9 (conf. 3), 6 (conf. 4) and 2 (one single impurity) scalar equations **. After evaluating the elements of $g$ from the static Green's tensor given in reference [16] the perturbative forces remain the only unknown quantities.

\section{Impurity Induced Perturbation Forces}

In accordance with (5) and (7) we split the perturbation forces into an electronic contribution

$$
\boldsymbol{f}_{m}^{\mathrm{e}}(\tilde{\boldsymbol{X}})=-\nabla_{\widetilde{\boldsymbol{X}}_{m}} \omega^{(2)}(\tilde{\boldsymbol{X}}, \boldsymbol{Y})
$$

and a protonic contribution

$$
\boldsymbol{f}_{m}^{\mathrm{p}}(\tilde{\boldsymbol{X}})=-\nabla_{\widetilde{\boldsymbol{X}}_{m}}\left\{\sum_{n} \sum_{k=1}^{2} \frac{Z e^{2}}{\left|\tilde{\boldsymbol{X}}_{n}-\boldsymbol{Y}_{k}\right|}+\frac{e^{2}}{\left|\boldsymbol{Y}_{1}-\boldsymbol{Y}_{2}\right|}\right\}
$$

\subsection{The protonic contribution}

Due to the equivalence of the interstitials and the fact that the reduced Green's matrices $g$ account for

** The construction of the reduced Green's matrix $g$ is shown representatively for configuration 2 in the Appendix. 
the influence of the second perturbation center, it is sufficient to calculate the force acting from one proton to an ion $m$ as

$$
\begin{aligned}
\boldsymbol{f}_{m}^{\mathrm{p}}(\tilde{\boldsymbol{X}}) & =-\nabla_{\tilde{\boldsymbol{X}}_{m}} \sum_{n=1}^{4} Z e^{2}\left\{\frac{1}{\left|\tilde{\boldsymbol{X}}_{n}-\boldsymbol{Y}\right|}-\frac{1}{\left|\boldsymbol{X}_{n}^{0}-\boldsymbol{Y}\right|}\right\} \\
& =\frac{Z e^{2}\left(\tilde{\boldsymbol{X}}_{m}-\boldsymbol{Y}\right)}{\left|\tilde{\boldsymbol{X}}_{m}-\boldsymbol{Y}\right|^{3}}
\end{aligned}
$$

The sum over $n$ denotes the change of the coulomb energy due to the displacements of the nearest neighbours. The value of the effective charge $Z$ will be discussed below.

\subsection{The electronic contribution}

This contribution to the perturbative forces is determined by the displacement dependence of the energy eigenvalue in (6). To calculate this small difference between the infinite electronic eigenvalues of the hydride system and the reference system we use the New Tamm-Dancoff-method. The two eigenvalue problems are treated within the formalism of quantum field theory and then transformed into a valence band representation. The filled valence band of the host crystal is defined as a "dressed" vacuum. A mapping into a tensor product space then yields a single functional eigenvalue equation for the difference between the two initial eigenvalues. This functional eigenvalue equation is equivalent to a Fock-space representation and so may be interpreted physically $[20,21]$.

In zeroth approximation, the procedure yields an eigenvalue equation for two excess electrons [15] described by the two-particle function $h\left(s_{\mathrm{i}}\right.$ and $\sigma_{\mathrm{i}}$ denoting the spin) ${ }^{* * *}$

$$
\begin{array}{rl}
\delta_{s_{1} \sigma_{1}} \delta_{s_{2} \sigma_{2}} & B\left(x_{1}, x_{2} \mid y_{1}, y_{2}\right) h\left(y_{1}, \sigma_{1}, y_{2}, \sigma_{2}\right) \\
= & \omega^{(2)} h\left(x_{1}, s_{1}, x_{2}, s_{2}\right)
\end{array}
$$

with an integral operator

$$
\begin{aligned}
& B\left(x_{1}, x_{2} \mid y_{1}, y_{2}\right) \\
& =S_{1}\left(x_{1}, y_{1}\right)\left[S_{1}\left(x_{2}, \boldsymbol{r}\right)\left\{\left(D+V_{\mathrm{p}}\right)\left(\boldsymbol{r}, \boldsymbol{r}^{\prime}, \tilde{\boldsymbol{X}}, \boldsymbol{Y}\right)\right\} S_{1}\left(\boldsymbol{r}^{\prime}, \boldsymbol{y}_{2}\right)\right] \\
& +S_{1}\left(\boldsymbol{x}_{2}, \boldsymbol{y}_{2}\right)\left[S_{1}\left(\boldsymbol{x}_{1}, \boldsymbol{r}\right)\left\{\left(D+V_{\mathrm{p}}\right)\left(\boldsymbol{r}, \boldsymbol{r}^{\prime}, \tilde{\boldsymbol{X}}, \boldsymbol{Y}\right)\right\} S_{1}\left(\boldsymbol{r}^{\prime}, \boldsymbol{y}_{1}\right)\right] \\
& +\operatorname{Tr}\left[V_{\mathrm{p}}(\boldsymbol{r}, \boldsymbol{Y}) S_{0}(\boldsymbol{r}, \boldsymbol{r})\right] S_{1}\left(\boldsymbol{x}_{1}, \boldsymbol{y}_{1}\right) S_{1}\left(\boldsymbol{x}_{2}, \boldsymbol{y}_{2}\right) \\
& +S_{1}\left(\boldsymbol{x}_{1}, \boldsymbol{r}\right) S_{1}\left(\boldsymbol{x}_{2}, \boldsymbol{r}\right) \frac{e^{2}}{\left|\boldsymbol{r}-\boldsymbol{r}^{\prime}\right|} S_{1}\left(\boldsymbol{r}^{\prime}, \boldsymbol{y}_{2}\right) S_{1}\left(\boldsymbol{r}, \boldsymbol{y}_{1}\right) .
\end{aligned}
$$

*** We use an extended Einstein convention, i.e. summation over multiple indices and integration over multiple arguments.
Here $\left(D+V_{\mathrm{p}}\right)$ is an abbreviation for the Hartree-Fockoperator

$$
\begin{aligned}
D\left(\boldsymbol{r}, \boldsymbol{r}^{\prime}, \tilde{\boldsymbol{X}}\right)= & {\left[-\frac{h^{2}}{2 m_{\mathrm{e}}} \Delta_{\boldsymbol{r}}+V_{\mathrm{I}}(\boldsymbol{r}, \tilde{\boldsymbol{X}})+e^{2} \int \mathrm{d} \boldsymbol{q} \frac{2 S_{0}(\boldsymbol{q}, \boldsymbol{q})}{|\boldsymbol{q}-\boldsymbol{r}|}\right] } \\
& \cdot \delta\left(\boldsymbol{r}-\boldsymbol{r}^{\prime}\right)+V_{\mathrm{ex}}\left(\boldsymbol{r}, \boldsymbol{r}^{\prime}\right),
\end{aligned}
$$

and the proton potential

$$
V_{\mathrm{p}}(\boldsymbol{r}, \boldsymbol{Y}) \delta\left(\boldsymbol{r}-\boldsymbol{r}^{\prime}\right)=-\sum_{k=1}^{2} \frac{e^{2}}{\left|\boldsymbol{r}-\boldsymbol{Y}_{k}\right|} \delta\left(\boldsymbol{r}-\boldsymbol{r}^{\prime}\right) .
$$

The operators $S_{0}$ and $S_{1}$ with $S_{0}+S_{1}=\mathbb{1}$ may be constructed with Bloch functions of the unperturbed crystal and project onto occupied $\left(S_{0}\right)$ and unoccupied $\left(S_{1}\right)$ states respectively. $S_{0}(\boldsymbol{q}, \boldsymbol{q})$ describes the electron density $n$ of the unperturbed host. Due to spin degeneracy we have $2 S_{0}(\boldsymbol{q}, \boldsymbol{q})=Z n(\boldsymbol{q})$. In our calculations we employ a density computed by K. M. Ho et al. using a selfconsistent pseudopotential theory [22]. Like these authors, we approximate the nonlocal exchange potential by a local Slater- $x_{\alpha}$-potential,

$$
\begin{array}{r}
V_{\text {ex }}\left(\boldsymbol{r}, \boldsymbol{r}^{\prime}\right)=-\alpha 3 e^{2}\left(\frac{3 Z n(\boldsymbol{r})}{8 \pi}\right)^{1 / 3} \delta\left(\boldsymbol{r}-\boldsymbol{r}^{\prime}\right), \\
\text { with } \quad \alpha=0.8 .
\end{array}
$$

$S_{1}$ orthogonalizes the eigenstates $\mid h>$ of (13) onto the filled valence band of the host. For practical purposes we take into account in $S_{1}$ only the $5 \mathrm{~s}$-electrons and describe them as plane waves,

$$
S_{1}\left(x, x^{\prime}\right)=\sum_{\boldsymbol{k} \text { unocc }} \frac{1}{\Omega_{0}} e^{i \boldsymbol{k}\left(x-x^{\prime}\right)} .
$$

In a further step we introduce orthogonality onto the localized states (core states and $4 \mathrm{~d}$ valence band states) by a hardcore added to the bare coulomb potential of the ions to obtain an effective potential

$$
V_{\mathbf{I}}(\boldsymbol{r}, \tilde{\boldsymbol{X}})=-Z e^{2} \sum_{n} \frac{1-e^{-\gamma\left|\boldsymbol{r}-\tilde{\boldsymbol{X}}_{n}\right|^{2}}}{\left|\boldsymbol{r}-\tilde{\boldsymbol{X}}_{n}\right|} .
$$

A hardcore parameter $0.4 \leq \gamma \leq 0.5$ reproduces the sand p-contribution of the pseudopotential of Ho et al. quite well. Within this range we fit the parameter to the experimentally obtained heat of solution and fix it to $\gamma=0.45$ [11]. This effective ion potential is split into two parts,

$$
V_{\mathrm{I}}(\boldsymbol{r}, \tilde{\boldsymbol{X}})=V_{\mathrm{I}}\left(\boldsymbol{r}, \boldsymbol{X}^{0}\right)+\left[V_{\mathrm{I}}(\boldsymbol{r}, \tilde{\boldsymbol{X}})-V_{\mathrm{I}}\left(\boldsymbol{r}, \boldsymbol{X}^{0}\right)\right] .
$$

The first term is evaluated together with the electronic potential $Z e^{2} \int \mathrm{d} \boldsymbol{q} \frac{n(\boldsymbol{q})}{|\boldsymbol{q}-\boldsymbol{r}|}$ (where $n(\boldsymbol{q})$ is taken from Ho 
et al.) in the reciprocal lattice. This yields the embedding energy of an excess electron into the unperturbed crystal

$$
V_{\mathrm{emb}}^{\mathrm{e}}\left(\boldsymbol{r}, \boldsymbol{X}^{0}\right)=Z e^{2} \frac{4 \pi}{\Omega_{0}} \sum_{k \neq 0} \frac{1-\hat{n}(\boldsymbol{k})}{|\boldsymbol{k}|^{2}} e^{i \boldsymbol{k} \boldsymbol{r}} .
$$

The second term accounts for the displacements of the next neighbouring ions. There is no term to describe the change of the electron density distribution around the diplaced ions. It is reasonable to assume that the localized $4 \mathrm{~d}$-electrons will follow the ions adiabatically and therefore lead to a screening effect with an effective charge $Z_{\text {eff }}$. We chose $Z_{\text {eff }}=5$ in all potentials which do not depend on the latice relaxation, all $4 \mathrm{~d}^{4}$ and $5 \mathrm{~s}^{1}$ are assumed to be valence electrons, and $Z_{\text {eff }}=1$ in all displacement dependent potentials (according to the displaced charge of the d-electrons). This approximation is also made when we calculate the embedding energy of the protons into the host. The displacement independent term is evaluated analogously to (21) in the reciprocal latice and the displacement dependent term is the sum over $n$ in (12) with $Z=1$ *.

The embedding energy of the protons in the valence band is described by

$\operatorname{Tr}\left[V_{\mathrm{p}}(\boldsymbol{r}, \boldsymbol{Y}) S_{0}(\boldsymbol{r}, \boldsymbol{r})\right]=-\sum_{k=1}^{2} e^{2} \int \mathrm{d} \boldsymbol{r} \frac{2 S_{0}(\boldsymbol{r}, \boldsymbol{r})}{\left|\boldsymbol{r}-\boldsymbol{Y}_{k}\right|}$

and appears as a renormalisation constant.

\subsubsection{A Separation Ansatz}

As the solution of the one-particle problem is known [11], it is natural to make a separation ansat7 to solve (13). For this purpose we expand the two-particle function $|h\rangle$ into Slater-determinants of the oneelectron functions. These functions are fourfold degenerate with respect to the spin and the localization at the first or the second interstitial. The operator (14) does not contain any explicit spin interaction, so we can separate the spin state and consider only the space-dependent part. We are left with a symmetric function, connected with an antisymmetric spin state (singlet) and an antisymmetric function, connected with three different symmetric spin states (triplet). Because of the orthogonality of these two functions we have two independent integro-differential equations for the symmetric and the antisymmetric function respectively.

* We discussed all the above mentioned approximations in greater details in [11].
The integral operator defined in (14) is uniquely decomposed into one-particle contributions $B_{1}$ and $B_{2}$, and a two-particle contribution $B_{12}$. Then these equations decompose into an equation for the oneelectron eigenvalue $\omega^{(1)}$,

$$
\left\langle h^{\mathrm{I}}(\boldsymbol{x})\left|B_{1}(\boldsymbol{x} \mid \boldsymbol{y})+B_{2}(\boldsymbol{x} \mid \boldsymbol{y})\right| h^{\mathrm{I}}(\boldsymbol{y})\right\rangle=2 \omega^{(1)},
$$

and an interaction potential

$\Delta \omega=\omega^{(2)}-2 \omega^{(1)}$

$=\frac{\left\langle h^{\mathrm{I}}(\boldsymbol{x})\left|B_{12}(\boldsymbol{x} \mid \boldsymbol{y})\right| h^{\mathrm{I}}(\boldsymbol{y})\right\rangle \pm\left\langle h^{\mathrm{II}}(\boldsymbol{x})\left|B_{12}(\boldsymbol{x} \mid \boldsymbol{y})\right| h^{\mathrm{I}}(\boldsymbol{y})\right\rangle}{1 \pm\left\langle h^{\mathrm{II}}(\boldsymbol{x}) \mid h^{\mathrm{I}}(\boldsymbol{x})\right\rangle}$,

where "+" denotes the singlet and "-" the triplet state respectively. $h^{\mathrm{I}}$ and $h^{\mathrm{II}}$ are product states of normalized one-particle eigenfunctions,

$$
\begin{aligned}
& h^{\mathrm{I}}(\boldsymbol{x})=h_{1}\left(x_{1}\right) h_{2}\left(x_{2}\right), \\
& h^{\mathrm{II}}(\boldsymbol{x})=h_{2}\left(x_{1}\right) h_{1}\left(x_{2}\right) .
\end{aligned}
$$

Although this spin valence method proposed first by Heitler and London [23] did not provide quantitatively accurate values for of the binding energy of the $\mathrm{H}_{2}$-molecule, we expect more reliable results. In our case there exists a lower limit on the distance between the protons given by the radius of the first shell of neighbouring interstitials $(1.17 \AA)$. This distance exceeds the bond length of $\mathrm{H}_{2}(0.74 \AA)$. It is well known that the results obtained by this procedure applied improve with increasing distance between the protons. Within the interesting range of ca. $1.1 \AA$ (Conf. 1)$2.3 \AA$ (Conf. 4) the error will be sufficiently small [24].

\section{One-particle Equation and Perturbation Forces}

Equation (23) separates into two uncorrelated oneparticle equations. For the solution of the one-particle problem we refer to [11]. In contrast to this former work we use a simplified expansion of the one-particle eigenfunction $h_{\mathrm{i}}(x)$,

$$
h_{i}(x)=\beta_{0} \Psi_{100}^{1}(x)+\sum_{n=1}^{7} \beta_{n} \Psi_{n 00}^{5}(x) .
$$

The generalized hydrogen functions $\phi_{n l m}^{z}$ are orthogonalized onto the valence band of the host,

$$
\Psi_{n l m}^{z}(\boldsymbol{x})=S_{1}(\boldsymbol{x}, \boldsymbol{r}) \phi_{n l m}^{z}(\boldsymbol{r}) .
$$

We neglect non-radial components, which can be shown to make a much smaller contribution [11]. With this expansion and the eigenvalue normalized by one term of (22) we transform the one-particle equation into an algebraic eigenvalue matrix equation. 
This equation depends on the radial displacement $\boldsymbol{s}^{\mathrm{rad}}$ of the next neighbours,

$$
\mathscr{M}\left(\boldsymbol{s}^{\mathrm{rad}}\right) \beta\left(\boldsymbol{s}^{\mathrm{rad}}\right)=\lambda^{(1)}\left(\boldsymbol{s}^{\mathrm{rad}}\right) \mathscr{U} \beta\left(\boldsymbol{s}^{\mathrm{rad}}\right) .
$$

We determine the eigenvalue $\lambda^{(1)}$ and the vector of coefficients $\beta_{n}^{z}$ as well as the one-particle electronic contribution to the perturbative forces (10),

$$
f_{m}^{e 1}(s)=-\nabla_{s_{m}} \omega^{(1)}(s)=-\frac{\partial}{\partial s_{m}^{\mathrm{rad}}} \lambda^{(1)}\left(s^{\mathrm{rad}}\right),
$$

within a range of 0.3 a.u. $\leq s^{\text {rad }} \leq 1$ a.u.; $m=1, \ldots, 4$ (next neighbours).

\section{Interaction Potential}

As a consequence of our representation for the projection operators $S_{1}(x, y),(18)$, they commute for distinct arguments. With the aid of this property and using that

$$
S_{1}(\boldsymbol{x}, \boldsymbol{y}) h_{\mathrm{i}}(\boldsymbol{y})=h_{\mathrm{i}}(\boldsymbol{x}), \quad \mathrm{i}=1,2,
$$

which holds in the zeroth approximation of the NTDprocedure [15] we can eliminate completely the $S_{1}$-projectors from the interaction term (24)**. The remaining contributions to the potential are in particular the coulomb interaction,

$$
\begin{aligned}
\mathscr{C}= & \iint \mathrm{d} \boldsymbol{r}_{1} \mathrm{~d} \boldsymbol{r}_{1}^{\prime} h^{*}\left(\boldsymbol{r}_{1}\right) h^{*}\left(\boldsymbol{r}_{2}^{\prime}\right) \frac{e^{2}}{\left|\boldsymbol{r}_{1}-\boldsymbol{r}_{1}^{\prime}\right|} h\left(\boldsymbol{r}_{2}^{\prime}\right) h\left(\boldsymbol{r}_{1}\right) \\
& -2 \int \mathrm{d} \boldsymbol{r}_{1} h^{*}\left(\boldsymbol{r}_{1}\right) \frac{e^{2}}{\left|\boldsymbol{r}_{1}-\boldsymbol{Y}_{2}\right|} h\left(\boldsymbol{r}_{1}\right)
\end{aligned}
$$

and the exchange interaction,

$$
\begin{aligned}
\mathscr{A}= & \iint \mathrm{d} \boldsymbol{r}_{1} \mathrm{~d} \boldsymbol{r}_{1}^{\prime} h^{*}\left(\boldsymbol{r}_{2}\right) h^{*}\left(\boldsymbol{r}_{1}^{\prime}\right) \frac{e^{2}}{\left|\boldsymbol{r}_{1}-\boldsymbol{r}_{1}^{\prime}\right|} h\left(\boldsymbol{r}_{2}^{\prime}\right) h\left(\boldsymbol{r}_{1}\right) \\
& -2 \int \mathrm{d} \boldsymbol{r}_{1} h^{*}\left(\boldsymbol{r}_{2}\right) \frac{e^{2}}{\left|\boldsymbol{r}_{1}-\boldsymbol{Y}_{2}\right|} h\left(\boldsymbol{r}_{1}\right) \cdot \int \mathrm{d} \boldsymbol{r}_{1} h^{*}\left(\boldsymbol{r}_{1}\right) h\left(\boldsymbol{r}_{2}\right) .
\end{aligned}
$$

The functions $h\left(\boldsymbol{r}_{1}\right)$ and $h\left(\boldsymbol{r}_{2}\right)$ are identical functions w.r.t. the interstitials 1 or 2 respectively.

An appropriate coordinate transform [25] results in $\boldsymbol{r}_{2}=\boldsymbol{r}_{2}\left(d, r_{1}\right)$ where $d$ is the distance between the interstitials. Hence an evaluation of the two-center integrals will be possible provided that we have a suitable representation of $h\left(\boldsymbol{r}_{2}\right)$ in terms of $d$ and $r_{1}$. Such representations are well known in molecular physics

** Clearly they are implicit in the eigenfunctions $h_{\mathrm{i}}(y)$ of the one-electron problem, since these are orthogonal to the Bloch states (18). and constructed by expanding these functions with respect to one centre into a series of spherical harmonics $\mathscr{Y}_{l}^{m}$ around the other one,

$$
\begin{aligned}
\left|h\left(\boldsymbol{r}_{2}\right)\right\rangle= & \sum_{l=0}^{\infty} \sum_{m=-l}^{l}\left|\mathscr{Y}_{l}^{m}\left(\vartheta_{1}, \varphi_{1}\right)\right\rangle \\
& \cdot\left\langle\mathscr{Y}_{l}^{m}\left(\vartheta_{1}, \varphi_{1}\right) \mid h\left(r_{2}, \vartheta_{2}, \varphi_{2}\right)\right\rangle .
\end{aligned}
$$

A formulation accomodated to numerically computed one-particle functions, which is relevant to our considerations, was presented by Löwdin [25]. He also pointed out a suitable convergence test, which allows us to fix the upper limit of our expansion to $l=12$.

Even though our calculations show the one-particle eigenvalue shifting considerably with the displacement of the nearest neighbouring ions, the eigenfunction within the interesting range is almost independent of the relaxation. As the only dependence of $\Delta \omega$ on $\tilde{\boldsymbol{X}}$ occurs via the eigenfunctions $h$, we set

$$
\boldsymbol{f}_{m}^{e^{2}}(\boldsymbol{s})=:-\nabla_{\widetilde{\boldsymbol{X}}_{m}} \Delta \omega(d, \tilde{\boldsymbol{X}}) \equiv 0 .
$$

Hence there are no contributions of the electron-electron interaction to the electronic perturbation forces.

We now define the electronic interaction to be the sum of the two-body contribution of the electronic energy and the bare coulomb interaction between the protons,

$$
\Delta W_{\mathrm{e}}(d)=: \frac{e^{2}}{d}+\Delta \omega(d)
$$

The numerically computed curve of this function is represented in Figure 4.

\section{Results: Storage Energy and Effective Interaction}

We are now in a position to carry out an iterative numerical solution of (9) using the perturbative forces given by

$$
\boldsymbol{f}_{m}=\frac{Z e^{2}}{\left|\tilde{\boldsymbol{X}}_{m}\right|^{2}}-\frac{\partial}{\partial \boldsymbol{s}_{m}^{\mathrm{rad}}} \lambda^{(1)}\left(\boldsymbol{s}^{\mathrm{rad}}\right),
$$

which result from a hydrogen center situated at the origin of the reference frame. With a starting vector $\boldsymbol{s}^{\text {rhs }}(0)=\mathbf{0}$ on the right hand side and an iteration procedure,

$$
s_{\mathrm{i}}^{\text {rhs }}(n)=\frac{s_{\mathrm{i}}^{\text {rhs }}(n-1)+s_{\mathrm{i}}^{\text {lhs }}(n)}{2}, \forall_{\mathrm{i}},
$$

we obtain a selfconsistent solution of (9) to an accuracy of $10^{-5}$ a.u. within less then 15 iterations. 


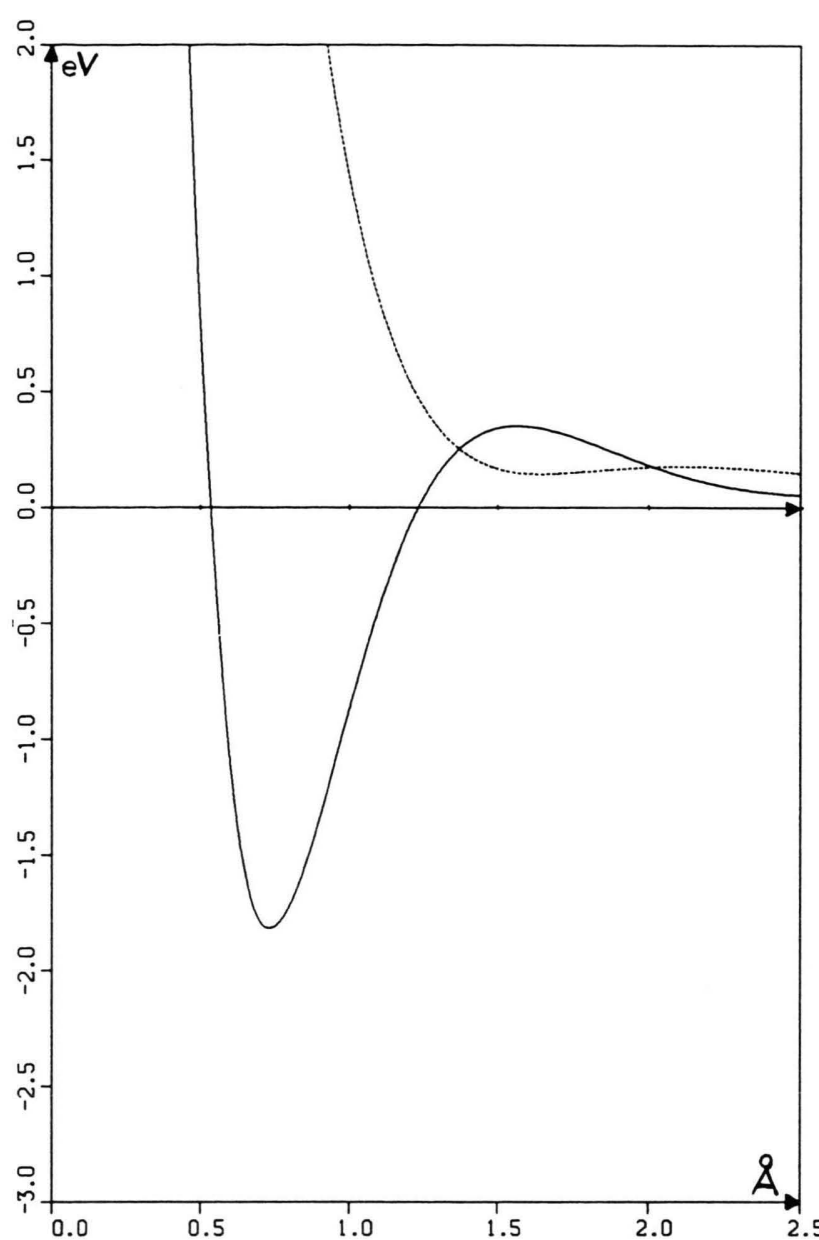

Fig. 4. Electronic interaction energy between two neighbouring H-centres. Solid curve: singlet electron state, dotted curve: triplet electron state.

Table 2. Interaction parameters of $\mathrm{H}$-centres neighbouring in first to fourth order on the interstitial lattice in niobium.

\begin{tabular}{llllc}
\hline & $\begin{array}{l}\text { Dis- } \\
\text { tance } \\
{[\AA]}\end{array}$ & $\begin{array}{l}\text { State of } \\
\text { Excess } \\
\text { Electrons }\end{array}$ & $\begin{array}{l}\text { Storage } \\
\text { Energy } \\
\Delta E_{s}[\mathrm{eV}]\end{array}$ & $\begin{array}{l}\text { Interaction } \\
\text { Energy } \\
W[\mathrm{eV}]\end{array}$ \\
\hline $\begin{array}{l}\text { Twice a } \\
\text { single centre }\end{array}$ & & & -5.026 & 0.000 \\
Config. 1 & \multirow{2}{*}{1.17} & singlet & -5.407 & -0.38 \\
& & triplet & -4.519 & 0.51 \\
Config. 2 & \multirow{2}{*}{1.65} & $\begin{array}{l}\text { singlet } \\
\text { triplet }\end{array}$ & -4.775 & 0.25 \\
& & singlet & -4.978 & 0.05 \\
Config. 3 & 2.02 & triplet & -4.918 & 0.11 \\
& & singlet & -4.999 & 0.11 \\
Config. 4 & 2.33 & triplet & -4.907 & 0.03 \\
& & & &
\end{tabular}

The displacements $s_{0}$ and the perturbation forces $f$ thus calculated determine the storage energy (1) of each configuration respectively,

$$
\begin{aligned}
\Delta E_{s}^{(2)}= & \frac{1}{2} \sum_{m} \boldsymbol{s}_{0 m} \boldsymbol{f}_{m}\left(\boldsymbol{s}_{0}\right)-\sum_{i=1}^{2} 5 e^{2} \frac{4 \pi}{\Omega_{0}} \sum_{k \neq 0} \frac{1-\hat{n}(k)}{|\boldsymbol{k}|^{2}} e^{i \boldsymbol{k} \boldsymbol{Y}_{i}} \\
& +\sum_{m} \sum_{i=1}^{2} e^{2}\left\{\frac{1}{\left|\tilde{\boldsymbol{X}}_{m}-\boldsymbol{Y}_{\mathrm{i}}\right|}-\frac{1}{\left|\boldsymbol{X}_{m}^{0}-\boldsymbol{Y}_{\mathrm{i}}\right|}\right\}+2 \lambda^{(1)}\left(\boldsymbol{s}_{0}\right) \\
& +\Delta W_{\mathrm{e}}^{(2)}\left(\left|\boldsymbol{Y}_{1}-\boldsymbol{Y}_{2}\right|\right)+2 \mathrm{Ryd} .
\end{aligned}
$$

The index $m$ runs over the ions participating in the relaxation. In particular, there are the following contributions to the storage energy:

- The elastic energy (4)

- Twice the embedding energy of one proton into the host analogous to (21)

- The change of this embedding energy due to the dislocation of the next neighbours of the protons (see (12))

- Twice the eigenvalue $\lambda^{(1)}\left(s_{0}\right)$ of the one electron problem (26) and

- The electronic interaction $\Delta W_{\mathrm{e}}(d)$ between the two hydrogen centres (32).

A comparison of the storage energy of each configuration to twice the storage energy of one $\mathrm{H}$-centre yields the interaction energy $W(2)$. A compilation of the results is given in Table 2 .

\section{Discussion and Conclusion}

We have calculated the effective interaction between two hydrogen centers in niobium, which are neighbouring from first to forth order. The elastic contribution has been treated in the classical harmonic approximation. We have employed a Born-Oppenheimer decoupling of the various subsystems and neglected vibration and diffusive motion of the host ions and the embedded protons. The electronic contribution has been evaluated by means of the NewTamm-Dancoff-method in zeroth approximation. This procedure reduces the electronic many-body problem in the crystal with two hydrogen impurities to a two-center and a two-excess electrons problem. We accounted explicitly for the dependence of the electronic eigenvalues on the lattice relaxation.

We find the effective interaction to be dependent on the distance between the H-centers and the spin state of their excess electrons. For the triplet state of this 
quasi-molecule the interaction is always repulsive, and for the singlet state we obtain an attractive interaction on the first coordination shell and repulsive interactions on the second, third and fourth shell. The ground state varies with the distance between singlet and triplet configuration: On the first shell we have a singlet ground state, on the second shell a triplet. On the third shell the singlet and the triplet state have the same energy (within the accuracy of our calculations), and on the fourth shell again the singlet has a lower energy.

The pure metal states, which are described by the projection operators $S_{0}$ and $S_{1}$ remain unchanged in zeroth approximation. This rigid band property excludes a description of the Friedel oscillations, which are typical around screened protons. A recent application of a higher order approximation of the NTD-procedure to the one-center problem removed the rigid band and lead to oscillations of the electron density around the zero level of the "dressed" vacuum in the vicinity of the embedded proton [26]. Band structure calculations on $\mathrm{H}$ in $\mathrm{Nb}$ show a hybridization of the pure metal states induced by the $\mathrm{H}$-impurities [29]. Within our approach this feature will emerge when we employ higher approximations of the theory.

A nonlocal change of the metal states, on the other hand, will demand a periodically loaded crystal (e.g. the $\beta$ - or $\gamma$-phase of $\mathrm{NbH}$ ), which is the starting point of band structure calculations. This indicates a second problem of our model: the restriction on two-body interactions can serve only as a first approach. Lattice relaxation and electron eigenvalues are genuine many-body problems and not additive in general. Further microsocpic investigations have to account for three- and more-particle correlations, i.e., they have to include models of three and more $\mathrm{H}$-centers in the host lattice $* * *$.

To check the validity of our calculations by a comparison with experimental results, we have to calculate the phase diagram of a lattice gas with the interaction parameters of Table 2 . In contrast to standard models we have to deal with 2 interaction parameters at each coordination shell up to the fourth one. We propose the construction of the grand partition function $\mathscr{Z}$ as follows.

\footnotetext{
*** An extensive numerical treatment of manybody potentials was performed by Oates and Stoneham [28], but they restricted on the elastic interactions.
}

Divide the total interaction $U_{\mathrm{ab}}$ between two hydrogen centers at interstitials $\mathrm{a}, \mathrm{b}$ into a short range contribution $W_{\mathrm{ab}}$ and a long range contribution $V_{\mathrm{ab}}$.

$$
U_{\mathrm{ab}}=\left\{\begin{array}{l}
W_{\mathrm{ab}},\left|\boldsymbol{Y}_{\mathrm{a}}-\boldsymbol{Y}_{\mathrm{b}}\right| \leq R_{0} \\
V_{\mathrm{ab}},\left|\boldsymbol{Y}_{\mathrm{a}}-\boldsymbol{Y}_{\mathrm{b}}\right|>R_{0} .
\end{array}\right.
$$

Chose the radius $R_{0}$ in such a way that the parameters of Table 2 account for the short range part. Let us consider the spin state by occupation variables $\tau$ which may take the values 0 for nonoccupation, +1 or -1 for spin up and spin down respectively. Now split the potential $W_{\mathrm{ab}}$ into a singlet and a triplet contribution with the conditions

$$
W_{\mathrm{ab}} \tau_{\mathrm{a}} \tau_{\mathrm{b}}=\left\{\begin{array}{l}
W_{\mathrm{ab}}^{\mathrm{s}}, \tau_{\mathrm{a}} \tau_{\mathrm{b}}=-1 \\
0, \tau_{\mathrm{a}} \tau_{\mathrm{b}}=0 \\
W_{\mathrm{ab}}^{\mathrm{T}}, \tau_{\mathrm{a}} \tau_{\mathrm{b}}=1 .
\end{array}\right.
$$

The long range elastic interaction $V_{\mathrm{ab}}$ may also include sample shape dependent contributions (for details see, e.g. [8]). We thus have to evaluate a configurational grand partition function

$$
\mathscr{Z}=\operatorname{Tr}\left[e^{-\beta\left(1 / 2 \sum_{\mathbf{a b}}\left(W_{\mathrm{ab}}+V_{\mathrm{ab}}\right) \tau_{\mathrm{a}} \tau_{\mathbf{b}}-\mu \sum_{\mathbf{a}} \tau_{\mathrm{a}}^{2}\right)}\right],
$$

where the trace is the sum over all possible configurations of occupation. Hamiltonians of this kind are familar from BEG-models or from lattice models of binary alloys with one magnetic component (see, e.g. [29]). Hence the direction for our further investigations on this topic is marked.

\section{Acknowledgement}

P. F. and K. H. are highly indebted to the Alfried Krupp von Bohlen und Halbach-Foundation for financial support. P. F. also likes to thank H. Fried for a critical reading of the manuscript.

\section{Appendix: Reduced Green's Matrices $g$ (Example)}

As Fig. 3 shows, we have 6 relevant ions in configuration 2 . The essential symmetries of the bond $\frac{a}{4}(2,1,0)$ $-\frac{a}{4}(2,3,0)$ are:

1. Mirror plane $x y$ formed by the ions 1, 2, 5 and 6 .

2. Mirror plant $y z$ formed by the protons I, II and the ions 3 and 4.

3. Mirror plane $x z$ formed by the ions 3 and 4 . 
The ions 1, 2, 5, and 6 are equivalent to one another w.r.t. the perturbation centres, the same holds for 3 and 4 . Thus we have only two distinct absolute values $f_{1}, f_{2}$ for the perturbative forces:

$$
\begin{aligned}
& \boldsymbol{f}_{1}=f_{1} \hat{\boldsymbol{e}}_{1}^{\mathrm{I}} ; \boldsymbol{f}_{2}=f_{1} \hat{\boldsymbol{e}}_{2}^{\mathrm{I}} ; \boldsymbol{f}_{3}=f_{2}\left(\hat{\boldsymbol{e}}_{3}^{\mathrm{I}}+\hat{\boldsymbol{e}}_{3}^{\mathrm{II}}\right) ; \\
& \boldsymbol{f}_{4}=f_{2}\left(\hat{\boldsymbol{e}}_{4}^{\mathrm{I}}+\hat{\boldsymbol{e}}_{4}^{\mathrm{II}}\right) ; \boldsymbol{f}_{5}=f_{1} \hat{\boldsymbol{e}}_{5}^{\mathrm{II}} ; \boldsymbol{f}_{6}=f_{1} \hat{\boldsymbol{e}}_{6}^{\mathrm{II}} .
\end{aligned}
$$

$\hat{e}_{i}^{j}$ are the unity vectors from proton $j$ to ion $i$. From (8) we get for $m=1, m=3$ :

$$
\begin{aligned}
\boldsymbol{s}_{m} & =\sum_{n=1}^{6} G_{m n} \boldsymbol{f}_{n}(\boldsymbol{s}) \\
& =f_{1}(s)\left(G_{m 1} \hat{\boldsymbol{e}}_{1}^{\mathrm{I}}+G_{m 2} \hat{\boldsymbol{e}}_{2}^{\mathrm{I}}+G_{m 5} \hat{\boldsymbol{e}}_{5}^{\mathrm{II}}+G_{m 6} \hat{\boldsymbol{e}}_{6}^{\mathrm{II}}\right)+f_{2}(s)\left[G_{m 3}\left(\hat{\boldsymbol{e}}_{3}^{\mathrm{I}}+\hat{\boldsymbol{e}}_{3}^{\mathrm{II}}\right)+G_{m 4}\left(\hat{\boldsymbol{e}}_{4}^{\mathrm{I}}+\hat{\boldsymbol{e}}_{4}^{\mathrm{II}}\right)\right] .
\end{aligned}
$$

[1] G. Alefeld and J. Völkl (eds.), Hydrogen in Metals, Vol. I \& II, Springer-Verlag Berlin, 1978.

[2] Y. Fukai and H. Sugimoto, Adv. Phys. 34, 263 (1985).

[3] A. Klamt and H. Teichler, phys. stat. sol. (b) 134, 533 (1986).

[4] C. K. Hall, in: P. Jena, C. B. Satterthwaite (eds.), Electronic Structure and Properties of Hydrogen in Metals, New York 1983.

[5] G. Alefeld, phys. stat. sol. 32, 67 (1969).

[6] H. Wagner and H. Horner, Adv. Phys. 23, 587 (1974).

[7] H. Horner and H. Wagner, J. Phys. C: Solid State Phys. 7, 3305 (1974)

[8] S. Dietrich and H. Wagner, Z. Phys. B36, 121 (1979).

[9] F. Wahl, R. Duscher, K. Göbel, and J. K. Maichle, Z. Naturforsch. 39 a, 524 (1984).

[10] W. P. Silin and W. J. Fainberg, Fortschr. Phys. 4, 233 (1956).

[11] K. Hau, P. Frodl, F. Sommer, and F. Wahl, Z. Naturforsch. 43a, 914 (1988).

[12] F. T. Sommer, K. Hau, P. Frodl, and F. Wahl, Z. Naturforsch. 43 a, 923 (1988)

[13] J. K. Nørskov, Phys. Rev. B20, 446 (1979).

[14] P. Nordlander, J. K. Nørskov, and F. Besenbacher, J. Phys. F: Metal Phys. 16, 1161 (1986).

[15] P. Frodl, Zur effektiven Wechselwirkung zweier Wasserstoffzentren in Niob, Thesis Tübingen 1988.
In matrix form we obtain (9)

$$
\left(\begin{array}{l}
\boldsymbol{s}_{1} \\
\boldsymbol{s}_{3}
\end{array}\right)=\left(\begin{array}{ll}
g_{11} & g_{12} \\
g_{31} & g_{32}
\end{array}\right)\left(\begin{array}{l}
f_{1}(s) \\
f_{2}(s)
\end{array}\right)
$$

with the vector-valued elements of the reduced Green's matrix $g$

$$
\begin{aligned}
& g_{m 1}=G_{m 1} \hat{\boldsymbol{e}}_{1}^{\mathrm{I}}+G_{m 2} \hat{\boldsymbol{e}}_{2}^{\mathrm{I}}+G_{m 5} \hat{\boldsymbol{e}}_{5}^{\mathrm{II}}+G_{m 6} \hat{\boldsymbol{e}}_{6}^{\mathrm{II}}, \\
& g_{m 2}=G_{m 3}\left(\hat{\boldsymbol{e}}_{3}^{\mathrm{I}}+\hat{\boldsymbol{e}}_{3}^{\mathrm{II}}\right)+G_{m 4}\left(\hat{\boldsymbol{e}}_{4}^{\mathrm{I}}+\hat{\boldsymbol{e}}_{4}^{\hat{I I}}\right) .
\end{aligned}
$$

[16] I. R. McGillivray and C. A. Sholl, J. Phys. F: Met. Phys. 13, 23 (1983).

[17] T. Schober, M. A. Pick and H. Wenzel, phys. stat. sol. (a) 18, 175 (1973).

[18] A. Klamt and H. Teichler, phys. stat. sol. (b) 134, 103 (1986).

[19] H. Peisl, X-Ray Studies of Interstitials in Metals, Proceedings of the Yamada Conference V, Kyoto 1982, North Holland Publ. Comp 1982, 41.

[20] W. Feist, Z. Naturforsch. 36a, 421 (1981).

[21] F. Wahl and W. Feist, Z. Naturforsch 36a, 429 (1981).

[22] K. M. Ho, S. G. Louie, J. R. Chelikovsky, and M. L. Cohen, Phys. Rev. B15, 1755 (1977).

[23] W. Heitler and F. London, Z. Phys. 44, 455 (1927).

[24] J. C. Slater, Quantum Theory of Matter. New York etc. 1968.

[25] P. O. Löwdin, Adv. Phys. 5, 1 (1956).

[26] K. Göbel and F. Wahl, Z. Naturforsch. 42a, 431 (1987).

[27] W. A. Oates and A. M. Stoneham, J. Phys. F: Met. Phys. 13, 2427 (1983).

[28] B. Dünweg and K. Binder, Phys. Rev. B36, 6935 (1987).

[29] H. I. Tao, K. M. Ho, and X. Y. Zhu, Phys. Rev. B34, 8394 (1986). 